\title{
Big Data, European Data Strategy, And Innovation: A Systematic Review of The Literature
}

\section{Cicero Eduardo WALTER ${ }^{1 *}$, Tiago VALENTE², Daniel Ferreira POLÓNIA³, Manuel AU-YONG- OLIVEIRA $^{4}$, Cláudia Miranda VELOSO ${ }^{5}$}

\author{
${ }^{1}$ Professor, M.Sc., Federal Institute of Education, Science and Technology of Piauí, Brazil; Ph.D. Student, University of \\ Aveiro, GOVCOPP, Aveiro, Portugal, E-mail: eduardowalter@ifpi.edu.br \\ 2 Ph.D. Student, University of Aveiro, Aveiro, Portugal, E-mail: tavalente@ua.pt \\ ${ }^{3}$ Professor, Ph.D., University of Aveiro, Department of Economics, Management, Industrial Engineering, and Tourism. \\ GOVCOPP, Aveiro, Portugal, E-mail dpolonia@ua.pt \\ ${ }^{4}$ Professor, Ph.D., University of Aveiro, Department of Economics, Management, Industrial Engineering, and Tourism. \\ GOVCOPP, Aveiro, Portugal, E-mail mao@ua.pt \\ ${ }^{5}$ Professor, Ph.D., University of Aveiro, ESTGA. GOVCOPP, Aveiro, Portugal, E-mail: cmv@ua.pt \\ ${ }^{*}$ Corresponding Author
}

\section{Received: 30.05.2021 Accepted: 03.08.2021 Published: 27.10.2021 DOI: 10.47750/QAS/22.184.02}

\begin{abstract}
As a result of the disruptive and comprehensive power of using Big Data, organizations need to commit to its application at a strategic level to achieve sustainable competitive advantages through its efficient use. Given this context and considering the importance of Big Data, this research aimed to identify how Big Data and its strategies, have been used to promote innovation, through a systematic review of the literature. Afterwards, it can be discussed how the research conducted on the subject can contribute to a greater chance of success for the European Data Strategy. From the research conducted in the Scopus and Web of Science databases, 25 titles related to the theme have been identified, 11 of which have been selected and read in full. The literature analyzed points out that the investigations have focused on developing models and strategies for the adoption of Big Data by companies, which commonly fail, due to not considering the needs and particularities of each business. In this sense, for the European Data Strategy to have greater chances of success, besides establishing an ecosystem propitious to the exploitation of the opportunities with the use of Big Data, it must promote the development of dynamic capacities. So that Small and Medium Enterprises can reconfigure their resources, routines and business models according to the use of data.
\end{abstract}

Keywords: Big Data; European Data Strategy; Innovation; Competitive Advantage; Systematic Literature Review.

\section{Introduction}

The analysis of a vast set of data can bring insights and innovations whose usefulness goes beyond the simple consumption experienced by a single individual, by generating benefits to society more broadly through the optimization of industrial systems, the improvement of public safety, or by understanding the environment (Cavoukian and Castro, 2014). In this sense, Big Data holds the promise of significantly reshaping everything from government to international economic development to the way basic science is done, based on the premise that data itself is a promising source of opportunities for innovation. However, many innovators do not clearly understand how Big Data can improve innovation processes (Gobble, 2013).

As a result of the disruptive and comprehensive power of using Big Data, organizations need to commit to its use strategically. Sustainable competitive advantages can come from developing and implementing actions, such as identifying market niches through Big Data, combining information from Big Data with emerging technologies to promote design-driven innovations, and using crowd innovation. Based on the assumption that the analysis of a wide range of consumer information can help develop new products and services (Morabito, 2015).
Given this context and considering the importance of Big Data, the purpose of this research is to identify how Big Data, and its strategies, have been used to promote innovation, through a systematic review of the literature. In a second moment, it is intended to discuss how the research conducted on the subject can contribute to the European Data Strategy having greater chances of success.

The justification for this research is based on the fact that Big Data can be considered a central factor of competitiveness for the development and growth of companies. As they can benefit from the analysis and management of a vast set of data, enabling opportunities for innovation management, innovation strategies or marketing innovations. Besides, traditional decisionmaking methods based on experience and intuition have changed dramatically to methods based on data analysis. In this sense, Big Data has played a significant role in predicting problems related to areas such as public health, economics, among others, so that decisions in these segments increasingly depend on the analysis of a vast set of data (Zhang et al., 2013).

Besides the present introduction, this investigation is structured into four sections. In the next section, the method used to achieve the established objectives is presented. Next, the results 
and discussion of the literature's systematic review are presented, constituting the central point of the present investigation and, finally, the conclusions and references used.

\section{Method}

The present investigation is characterized as a review of the literature. In this way, it started from selecting documents available on a particular topic, bringing in their essence information, ideas, data, or evidence from a specific point of view to achieve certain objectives or express a statement on the nature of a particular topic and how it has been investigated (Hart, 2018). It consists of a systematic review, as it uses rigorous criteria to identify, critically evaluate, and synthesize all the available literature on a given subject (Fink, 2019).

In this sense, the methodological design used is expressed in 3 distinct phases, namely in (I) Planning and Selection, (II) Extraction and (III) Execution, as recommended by some authors (Cronin et al., 2008; Okoli and Schabram, 2010) for the effective conduct of a systematic literature review.

In phase I, Planning and Selection, the literature review's purposes were defined from the following research questions: how has Big Data, in terms of applications and data strategies, been used to promote innovation? What lessons can be learned to make the European Data Strategy more successful?

Still, in this first phase, Scopus and Web of Science databases were selected due to their relevance in scientific publications. These databases are the most widely used by different fields of studies for literature research (Guz and Rushchitsky, 2009) and research and localization in the literature of information resources, establishing, as inclusion criteria, studies linked to the areas of Management, Economics, and Accounting.
The Boolean operators used were "Big Data" AND "Data Strategy" AND "Innovation", whose results are expressed in

Table 1. As a result of the research, 25 titles were found (i.e. 18 in Scopus and 7 in Web of Science). However, after reading the 7 titles from the Web of Science, it was found that 3 were repeated in Scopus and the one remaining did not fit the parameters defined for inclusion, being from the Health area, so they were excluded. From reading the 18 titles of the articles found at Scopus, 8 were selected for the reading of the abstract, whose criterion for selection was the exclusive focus on Big Data and Big Data strategies. Then, all 8 articles were selected and read in their entirety, considering that they met the inclusion criteria initially proposed.

Concerning phase II, Extraction, a qualitative approach was chosen to extract information, since this approach allows the development of a deeper understanding of events and situations (Cooper and Schindler, 2014). Regarding phase III, Execution, the information was analyzed employing a meta-synthesis, defined as a non-statistical technique used to integrate, evaluate and interpret the results of multiple studies (Cronin et al., 2008). Above all, through the analysis and synthesis of key elements of each of the studies, which were later grouped into a conceptual block due to their similarities, renamed "Models and Strategies employed through the use of Big Data", constituting part of the results and discussion section.

In general terms, about the purposes of the literature review conducted (Hart, 2018), this research aims to present an analytical and critical assessment of the subject in question. So it can distinguish what has been done and what is still possible in terms of research, as well as to establish a general context in which it is possible to rationalize the significance of the subject under analysis.

\begin{tabular}{|l|l|lc|lc|l|l|}
\hline Databases & Boolean operators & \multicolumn{2}{|l|}{ Titles } & \multicolumn{2}{|l|}{ Abstracts } & \multicolumn{2}{l|}{ Papers } \\
\hline & & Total & Accepted & Total Accepted & Total Accepted \\
\hline Scopus & $\begin{array}{l}\text { "Big Data" AND "Data } \\
\text { Strategy" AND "Innova- } \\
\text { tion" }\end{array}$ & 18 & 8 & 8 & 8 & 8 & 8 \\
\hline Web of Science & $\begin{array}{l}\text { "Big Data"AND "Data } \\
\text { Strategy" AND "Innova- } \\
\text { tion" }\end{array}$ & 7 & 3 & 3 & 3 & 3 & 3 \\
\hline Total & & 25 & 11 & 11 & 11 & 11 & 11 \\
\hline
\end{tabular}

Table 1: Search Results in Selected Databases and Articles.

\section{Results and Discussion}

Models and Strategies employed through the use of Big Data

Most organizations fail to implement Big Data projects due to a lack of alignment with strategic organizational objectives and failure to consider the necessary organizational capabilities. Jedlitschka (2017) presents a conceptual model to assist in the implementation of Big Data projects, as well as in the development of strategies for the same. Business innovations based on Big Data must start from considering the current context of a given organization, especially concerning its business needs, the potential business and Big Data solutions. Therefore to determine the potential benefits, methods and organizational capabilities for adopting Big Data, including finally a process of evaluation and readiness to implement the idealized solutions.

In addition to the model mentioned above, which combats the lack of alignment with strategic objectives and the lack of consideration of capacities by organizations, Mazzei and Noble (2017) claim that Big Data improves the functional capabilities of an organization. The model proposed by these authors provides an appropriate basis for internal corporate strategy discussions involving investments in Big Data, explaining how firms create value through various approaches. Thus, they present a 3 layers model, namely: 1. Big Data as a tool in the traditional value chain; 2. Big Data as a stimulus for new ventures and industry development; 3. Big Data as a driver of competitive strategy. In short, it reveals how more proactive organizations leverage Big Data initiatives to develop and maintain competitive advantage at various levels.

Accordingly, and considering the growing technological dependence and, in turn, in the data, according to Al-Qirim et al. (2017), this competitive advantage can be generated because 
Big Data strategies are disruptive. It can also challenge and foster the need for organizations to use this technology more effectively and ethically through a Big Data adoption model that allows detecting key predictors of success in its two aspects, technology, and management. Thereby highlighting different contributions, implications, and recommendations regarding the alignment between the organizational strategy and its needs according to a broad set of data.

In the same sense, intending to develop and empirically test a conceptual model for the Big Data strategy, Lakoju and Serrano (2017) using a case study conducted at a Nigerian radio station. They have found evidence that the model called SAVI$B I G D$, developed through interviews and focus groups with the organization's main stakeholders - consisting of 5 phases that seek an assessment of the current state of the organization, as well as solutions in terms of Big Data - allows the alignment of business strategies with Big Data projects, bringing benefits such as obtaining competitive advantages while keeping costs at low levels. In a first moment, the model developed allows the continuous generation of strategic objectives of Big Data that are aligned with the organizational needs, which will later be treated by the developers of the Big Data solution.

According to Kabir and Carayannis (2013), gaining a competitive advantage through business analytics and intelligent solutions is still a developing thing for many organisations. However, they understand that there is a wide range of knowledge and insights that can be extracted by using Big Data, considered as tacit knowledge that once removed, becomes explicit and widely available to an organization. Accordingly, these authors present a conceptual model for developing Big Data strategies that bring competitive advantages based on 3 distinct and sequential phases. According to the authors, the Big Data initiative must start from the satisfaction of prerequisites (Phase 1) related to management support, infrastructure, people, data-based culture, and absorptive capacity. Then, processing (Phase 2) describes the establishment of objectives, team building, metrics, and implementation plans to understand how the combination of people, processes, and technologies influences organizational performance. Finally, the authors argue that the outputs (Phase 3 ) in terms of human capital, innovation, and new knowledge lead to competitive advantages.

In a similar way to the authors mentioned above, Chen et al. (2016) point out that Big Data as a service has been a viable alternative to solve a series of problems in the implementation of a Big Data strategy, among which are the complexity of the systems, talent shortage, and high development and implementation costs. As a result of this scenario, when analyzing the Cisco Intercloud Analytical Platform, based on a model called "NeoMetropolis" - characterized by the use of different open source technologies that facilitate integration, easy prototyping for the expansion of choices in a single platform, enabling organizational innovation -, the referred authors found evidence that the adoption of Big Data as a service not only solves the main problems of adopting a Big Data strategy but also allows the promotion of innovation by customers in a more economical way through Big Data.

Adding to that, Trabucchi et al. (2018) investigating the distinctive features of user-generated Big Dates (UGBD), as well as the way they favor the development of value creation strategies, using multiple case studies on social media applications used in smartphones (Twitter, Spotify, Strava and Deliveroo), found evidence that user-generated Big Dates can be defined more as a user-centered innovation tool (UCl) than a user-driven innovation tool (UDI), as users promote insights into their behaviors and needs, rather than promoting solutions in a co-creation process, characteristic of the user-driven innovation approach. Also, the authors point out that the Big Data generated by users enable the creation of value through different types of innovation related to users, the ecosystem of partners from a service perspective and, by opening up to new customers from a business model innovation perspective, contributing to Open Innovation (OI) both in terms of inbounds (i.e. user insights for service improvements) and outbound (i.e. information on preferences and behaviors provided to partners that enable the development of innovations from this data).

Intending to develop and present a model to support an entrepreneurial plan based on sensors and the development and mapping of new products and services, Brown (2017) also addresses data from users. In this sense, the author defines different data source clusters, which he calls crowdsourcing. These techniques are (i) situated crowdsourcing (digital elements in everyday environments), (ii) spatial crowdsourcing (temporal dimensions), (iii) crowdsensing (autonomous sensors, positioned in the environment or embedded in hardware devices, functioning as a source of data related to mobility) and (iv) crowdsourcing through personal use devices (sensors embedded in the hardware of devices connected to the human body, clothing or accessories). Additionally, it discusses a model that allows organizing sensor-based entrepreneurship opportunities by two characteristics: level of data aggregation - it should be determined how to combine the data created by sensors - and sensor location - it should be determined where to locate the sensors that provide the data. The author concludes the study by reflecting that all this new data will change the way companies interact with their customers, as this data can help them build stronger and more personal relationships.

In terms of the breadth possible in implementing Big Data strategies, Lacam (2020) examines the interdependence of relational strategy and SMEs' data management policies during product innovation. Its model combines three theoretical dimensions: the characteristics of Big Data policies, an innovative product, and a relational strategy. The role of Big Data in the creation of new products can be explained by the need for SMEs to explore a broad spectrum of new information that was traditionally far from the company's field of expertise. Thus they can better capture developments in current or future markets, to shake up the market and make existing products obsolete.

Still, on the said amplitude, Sun et al. (2020) also tell us about the legislative context factors. The authors' study presents a model that incorporates factors from the technological, organizational, and legislative context, influencing an organization's decision to adopt Big Data strategies. The results indicate that relative advantage, technological competence, technological resources, support for top management, competitive pressure, and the legislative environment positively influence Big Data's adoption by organizations. As main results, the authors showed that technological competence leads to greater confidence of executives in decision making, namely to adopt new information technology. The "top management support" factor was revealed to significantly affect the adoption of Big Data strategies. Regarding the size of the organization, it is revealed that this factor does not significantly impact the adoption of Big Data.

Finally, to complete this discussion regarding the models and strategies employed through the use of Big Data, and the number of digital businesses is increasing, Unhelkar and Askren (2020) present a Big Data model for "agile" organizations - Big Data Framework for Agile Business (BDFAB). It is a strategic model that integrates: values, functions, phases, artifacts, practices, and business parameters. This model has a direct and practical impact on risk reduction in digital business transformation, using Big Data in organizations of medium and/or large size, which need a multidisciplinary, holistic, and long-term approach. Additionally, this model allows identifying and incorporating key values of the organization in a Big Data strategy.

The European Data Strategy: what lessons can be drawn from the literature? 
In a largely knowledge-based economy, the proper use of data can bring opportunities in different sectors such as transportation, health, or manufacturing. Through improvements in data processing and analysis, more specifically by Big Data, it will be possible to transform the European service industry by adding value at different stages of the data value chain, resulting in the creation of a broad set of innovative products and services based on the use of information (European Union, 2021).

Against this background, the European Union, through the European Data Strategy (European Commission, 2020), recognizes that to materialize this scenario, it is necessary to provide Small and Medium Enterprises with infrastructures, data services, and cloud computing that have essential functionalities regarding security, sustainability, interoperability, and scalability. European companies can benefit from the entire value chain, encompassing the generation, processing, access and reuse of data in their activities to promote innovation.

In this sense, this strategy brings as practical actions the development of an investment route that will count with public and private agents for the creation of common platforms. It will enable Small and Medium Enterprises to access a wide variety of cloud computing services for secure data storage and sharing, applications that range from artificial intelligence to simulation, modeling, digital twins (i.e. virtual replicas of products, processes or physical systems) and high-performance computing resources, as well as actions to strengthen the capabilities of these companies to take advantage of opportunities offered by data-based business models, such as legal advice and incentive programs for the development of data-based services in incubation, through the Horizon Europe and Digital Europe programs (European Commission, 2020).

However, although the European Data Strategy brings in its essence a set of actions that allow a greater understanding of the legal context, service availability, access infrastructures to a broad set of data and incentive programs for the development of data-based services in an incubation regime, allowing Small and Medium Enterprises to have access to a set of new information that they would not otherwise have, being following what is advocated by some authors (Chen et al., 2016; Lacam, 2020; Sun et al., 2020), for an adequate Big Data strategy, it should be emphasized that it will be necessary to go beyond that.

In a more specific way, as evidenced by several authors (AlQirim et al., 2017; Jedlitschka, 2017; Kabir \& Carayannis, 2013; Lakoju \& Serrano, 2017; Mazzei \& Noble, 2017; Unhelkar \& Askren, 2020), the adoption of Big Data must be aligned with the strategic needs and particular characteristics of each business, otherwise Big Data initiatives will not bring the expected benefits. From this perspective, for the European Data Strategy to have a greater chance of success, it must consider not only providing an ecosystem conducive to seizing opportunities with the use of Big Data but also fostering the development of dynamic capabilities (Teece et al., 1997; Winter, 2003; Zahra et al., 2006). Thus, Small and Medium Enterprises can reconfigure their resources, routines and business models based on data to achieve new ways and innovative sources of competitive advantage.

\section{Conclusion}

The systematic review of the literature carried out in this work allowed us to identify the assumptions and considerations to be adopted in a Big Data implementation strategy, strategies that are increasingly required by organizations to foster their development in an increasingly challenging market context, as well as to point ways for the European Data Strategy to have greater chances of success.

The models found for the implementation of Big Data strategies are distinct, however, globally, all focus on one goal, develop the organization and achieve a competitive advantage (Al-
Qirim et al., 2017; Chen et al., 2016; Jedlitschka, 2017; Kabir and Carayannis, 2013; Lakoju and Serrano, 2017; Mazzei and Noble, 2017)

Many organizations fail to implement Big Data projects due to a lack of alignment with strategic organizational objectives and lack of consideration of the required capabilities (Jedlitschka, 2017). Still, Mazzei and Noble (2017) reinforce that through strategic discussions involving investments in Big Data it is possible to create value, because the Big Data implementation strategy is an approach that will make the company improve organizational capabilities, at the level of the value chain, new investments and corporate strategy, which is corroborated by Lakoju and Serrano (2017), that in other words, they argue that the implementation of Big Data allows the alignment of different business strategies with the Big Data projects.

To Lakoju and Serrano (2017) and Mazzei and Noble (2017) will undoubtedly be a way to achieve a competitive advantage. Big Data's strategy cannot be neglected, because there is a constant increase in dependency on technology. According to AlQirim et al. (2017), implementing a Big Data strategy will make organizations disruptive and challenging, fostering the need for them to use this technology more effectively and ethically.

Following what has been said in the previous paragraphs, and still, in the context of implementing Big Data strategies, some authors are more comprehensive in their studies, or, address other more specific topics (Brown, 2017; Lacam, 2020; Sun et al., 2020; Trabucchi et al., 2018; Unhelkar and Askren, 2020).

Trabucchi et al. (2018) introduce the concept of data from users, which can be defined more as a user-centered innovation tool, as users promote insights into their behavior and needs. Brown (2017) presents us with 4 different data source clusters, which it calls crowdsourcing: situated, temporal, autonomous sensors, and through personal use devices. Arguing that all this new data will change the way companies interact with their customers, as this data can be used to help them build stronger and more personal relationships.

Still in the context of possible specificities in the application of Big Data strategies, Lacam (2020) tells us about the implementation during the innovation of new products in SMEs, which allow exploring a wide spectrum of new information that was traditionally distant from the field of specialization of the company, to better capture the developments of current or future markets, to shake up the market and make existing products obsolete.

Sun et al. (2020) present an implementation model incorporating 3 factors, technological, organizational, and the legislative context, the latter being a more unconventional approach. Their study proved that the legislative environment positively influences the adoption of Big Data by organizations.

The number of digital businesses is increasing, Unhelkar and Askren (2020) present a strategic model for "agile" companies (rapid adaptation) that has a direct and practical impact in reducing risks in the transformation of digital business. Most organizations fail to implement Big Data projects, however, the adoption of strategic models, such as those presented, allow the consolidation of the organization's strategy and enhance its organizational capabilities. It is these models that bring contributions, implications, and recommendations to organizations. All these new ways of dealing with this new data will change the way companies interact with their clients, as this data can also help them build stronger and more personal relationships. Thus, organizations can leverage competitive advantage and stay current in increasingly dynamic and existing markets. It is essential to make clear that the size of organizations has nothing to do with the need or success in implementing this technology.

As far as the European Data Strategy is concerned, it can be seen that it is premised on the establishment of an ecosystem conducive to seizing opportunities through the use of Big Data, 
focusing primarily on the development of services and infrastructure, as well as legal advice, which allows Small and Medium Enterprises to develop new products and innovative services based on information. However, for this strategy to achieve the potential of the European Union to be successful in the objective of economic development based on data, it is imperative that it also encourages the development of dynamic capabilities so that Small and Medium Enterprises can reconfigure their resources, routines and business models according to the use of data to achieve new ways and innovative sources of competitive advantage.

The present research presents as limitations the fact that there is no information or research available on the results of the effective implementation of the European Data Strategy, so the recommendations expressed herein are based on studies for the adoption of Big Data conducted in a micro context.

As future investigations, it is suggested to evaluate which organizational factors in different sectors of the economy most influence Big Data's adoption and how dynamic capabilities can enhance Big Data strategies.

\section{References}

[1] Al-Qirim, N., Tarhini, A., \& Rouibah, K. (2017). Determinants of Big Data Adoption and Success. Proceedings of the International Conference on Algorithms, Computing and Systems - ICACS '17. doi:10.1145/3127942.3127961

[2] Brown, T. E. (2017). Sensor-based entrepreneurship: A framework for developing new products and services. Business Horizons, 60(6), 819-830. doi:10.1016/j.bushor.2017.07.008

[3] Cavoukian, A., \& Castro, D. (2014). Big data and innovation, setting the record straight: de-identification does work. Information and Privacy Commissioner Ontario, Canada.

[4] Chen, H.-M., Kazman, R., Haziyev, S., Kropov, V., \& Chtchourov, D. (2016). Big Data as a Service: A Neo-Metropolis Model Approach for Innovation. 2016 49th Hawaii International Conference on System Sciences (HICSS). doi:10.1109/hicss.2016.674, $5458-5467$.

[5] Cooper, D. R., \& Schindler, P. S. (2014). Business Research Methods: Vol. twelfth edition. McGraw-Hill/Irwin.

[6] Cronin, P., Ryan, F., \& Coughlan, M. (2008). Undertaking a literature review: a step-by-step approach. British Journal of Nursing, 17(1), 38-43. doi:10.12968/bjon.2008.17.1.28059

[7] European Commission. (2020). A European Strategy for Data (COM(2020) 66 final). European Commission.

[8] European Union. (2021, January 11). Shaping Europe's Digital future: policies, Big Data. European Commission. https://ec.europa.eu/digital-single-market/en/big-data

[9] Fink, A. (2019). Conducting Research Literature Reviews: From the Internet to Paper. SAGE Publications.

[10] Gobble, M. M. (2013). Big Data: The Next Big Thing in Innovation. Research-Technology Management, 56(1), 64-67. doi: $10.5437 / 08956308 \times 5601005$

[11] Guz, A. N., \& Rushchitsky, J. J. (2009). Scopus: A system for the evaluation of scientific journals. International Applied Mechanics,
45(4), 351-362. doi:10.1007/s10778-009-0189-4

[12] Hart, C. (2018). Doing a Literature Review: Releasing the Social Science Research Imagination. Sage Publications.

[13] Jedlitschka, A. (2017). Analyzing the Potential of Big Data. Lecture Notes in Computer Science, 613-616. doi:10.1007/978-3319-69926-4 52

[14] Kabir, N., \& Carayannis, E. (2013). Big Data, Tacit Knowledge, and Organizational Competitiveness. Proceedings of the 10th International Conference on Intellectual Capital, Knowledge Management, and Organisational Learning (ICICKM-2013), 220-227.

[15] Lacam, J.-S. (2020). Data: A collaborative? The Journal of High Technology Management Research, 31(1), 100370. doi:10.1016/j.hitech.2020.100370

[16] Lakoju, M., \& Serrano, A. (2017). Saving costs with a big data strategy framework. 2017 IEEE International Conference on Big Data (Big Data). doi:10.1109/bigdata.2017.8258188, 2340-2347.

[17] Mazzei, M. J., \& Noble, D. (2017). Big data dreams: A framework for corporate strategy. Business Horizons, 60(3), 405-414. doi:10.1016/j.bushor.2017.01.010

[18] Morabito, V. (2015). Big Data and Analytics for Competitive Advantage. Big Data and Analytics, 3-22. doi:10.1007/978-3-31910665-6_1

[19] Okoli, C., \& Schabram, K. (2010). A Guide to Conducting a Systematic Literature Review of Information Systems Research. SSRN Electronic Journal. doi:10.2139/ssrn.1954824, 1-50.

[20] Sun, S., Hall, D. J., \& Cegielski, C. G. (2020). Organizational intention to adopt big data in the B2B context: An integrated view. Industrial Marketing Management, 86, 109-121. doi:10.1016/j.indmarman.2019.09.003

[21] Teece, D. J., Pisano, G., \& Shuen, A. (1997). Dynamic Capabilities and Strategic Management. Strategic Management Journal, 18(7), 509-533.

[22] Trabucchi, D., Buganza, T., Dell'Era, C., \& Pellizzoni, E. (2017). Exploring the inbound and outbound strategies enabled by user generated big data: Evidence from leading smartphone applications. Creativity and Innovation Management, 27(1), 42-55. doi:10.1111/caim.12241

[23] Unhelkar, B., \& Askren, J. (2019). Development and Initial Validation of the Big Data Framework for Agile Business: Transformational Innovation Initiative. Advances in Information and Communication, 952-960. doi:10.1007/978-3-030-12388-8_65

[24] Winter, S. G. (2003). Understanding dynamic capabilities. Strategic Management Journal, 24(10), 991-995. doi:10.1002/smj.318

[25] Zahra, S. A., Sapienza, H. J., \& Davidsson, P. (2006). Entrepreneurship and Dynamic Capabilities: A Review, Model and Research Agenda*. Journal of Management Studies, 43(4), 917955. doi:10.1111/j.1467-6486.2006.00616.x

[26] Jinsong Zhang, Yan Chen, \& Taoying Li. (2013). Opportunities of innovation under challenges of big data. 2013 10th International Conference on Fuzzy Systems and Knowledge Discovery (FSKD). doi:10.1109/fskd.2013.6816280, 669-673. 